\title{
Telegram as An Immigration Management Tool
}

\section{Sarah Nikkhah}

Indiana University - Purdue

University Indianapolis (IUPUI)

Indianapolis, IN 46202, USA

snikkhah@iu.edu

\section{Andrew D. Miller}

Indiana University - Purdue University Indianapolis (IUPUI)

Indianapolis, IN 46202, USA

andrewm@iu.edu

\section{Alyson L. Young}

Indiana University - Purdue

University Indianapolis (IUPUI)

Indianapolis, IN 46202, USA

youngaly@iupui.edu

Permission to make digital or hard copies of part or all of this work for personal or classroom use is granted without fee provided that copies are not made or distributed for profit or commercial advantage and that copies bear this notice and the full citation on the first page. Copyrights for third-party components of this work must be honored. For all other uses, contact the Owner/Author.

CSCW '18 Companion, November 3-7, 2018, Jersey City, NJ, USA

(C) 2018 Copyright is held by the owner/author(s).

(C) 2018 Copyright is held by the owner/a

https://doi.org/10.1145/3272973.3274093

\begin{abstract}
This paper describes an ongoing study that examines Iranian immigrants' use of communication tools and online communities throughout the immigration process. We conducted observations on 30 Iranian immigration-related groups on the Telegram messaging application to understand its impacts on immigrants' collaboration, information-seeking, and informationsharing behavior. This research has implications to support immigration practices through technology.
\end{abstract}

\section{Author Keywords}

Immigration; Online Communities; Iran; Telegram

\section{ACM Classification Keywords}

Human-centered computing User studies: Humancentered computing Empirical studies in $\mathrm{HCI}$ : Human-centered computing Collaborative and social computing

\section{Introduction}

Immigrants face a wide range of challenges including language barrier, finding a job, getting acquainted with the new system [6]. Immigrants seek out other people with similar experiences who can offer guidance, solidarity, and information. This is especially important for Iranian immigrants to the US. Static resources found through search results may quickly become out of date due to the dynamic and fast-changing political 


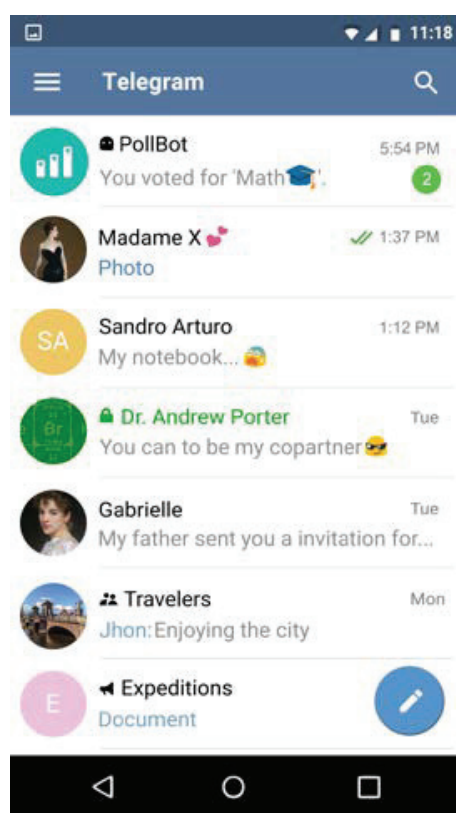

Figure 1: The Telegram Android interface (source: Telegram Messenger LLP via Wikimedia Commons) situations in both countries. In this work, we observed how Iranian immigrants to the US use Telegram to seek out information and connect with each other throughout the immigration journey.

\section{Related work: Telegram and Iran}

Over the last few years, Telegram has become one of the most used messaging applications in Iran. Its high speed and availability across operating systems combined with its secure transmission protocol led to broad adoption. Although recently filtered by the Iranian government [3], Telegram remains popular with Iranians planning or considering immigration to the US. Researchers have begun to examine Telegram, including research in or about Iran, but there are few studies of its impact on immigration. For example, Daraghi Nobari et al. detected advertisement and spam messages by crawling Telegram public data and classifying messages [2]. Xodabande examined the effectiveness of using Telegram in teaching English language pronunciation to Iranian EFL learners [5]. Ghaffari et al studied the effect of using Telegram for as a public health intervention for breastfeeding children among Iranian women [4]. Overall, there is still not enough research which examines the role of ICTs on information behaviors of immigrants [1].

\section{Methods}

From January to May 2018 we observed 30 public Telegram groups and channels focused largely on the processes of moving to the US from Iran, either permanently or for educational reasons. These groups are used to discuss matters related to the different stages of immigration. Conversations focus on a wide range of topics including the visa application process, peoples' experiences with the embassy interview, making plans to travel together to the embassy, and seeking and sharing information about moving to the US. Observations involved searching for topics using different Persian keywords and hashtags on topics such as "Embassy Interview", "Bank Account", "Job", "Car", "Medical Insurance", "Shopping", "Diversity Visa" and documenting important topics as well as capturing examples in screenshots. The data were analyzed using open coding to identify prominent themes regarding immigrating to the US. In this process, we observed individuals appropriating Telegram features to work around limitations of the technology. Next, we provide examples of how Iranians are using Telegram to get their immigration information needs and the rules and workarounds they have created to overcome the limitations of Telegram. The keywords and hashtags that we searched for and the example messages are all translated from Persian to English.

\section{Finding community: channels, 'supergroups', and links}

On Telegram, Iranian immigrants meet in public 'channels', where an administrator can broadcast information, 'Supergroups', which are large group chats with up to 100,000 members, and traditional chat groups. Unlike traditional forums, there is no official directory or in-app search mechanism to find topicbased groups or channels. Since Telegram does not have a search feature to locate relevant groups, individuals must either know the exact channel link to locate it or be added by the admin. In this section, we describe how Iranian immigrants to the US use channels, supergroups, and links to connect with each other during the immigration process. 


\section{A Supergroup related to the USA Visa runs a bot which posts this to the group and deactivates the group; therefore, no one can send any messages:} "The group automatically turns off until six o'clock in the morning. Use the opportunity and study the previous conversations"

Textbox 1: An instance of the use of bots in supergroups to prevent sending messages at the inconvenient time.

\section{One of the main rules of a supergroup related to student application is: "Any type of advertising, sharing the link to other groups, channels, and irrelevant topics will be treated differently and, if repeated, the presence of the violator in the group will be prevented."}

Textbox 2: An example of a rule pinned to the supergroup by the admin.

\section{Channels}

Channels are optimized to share information to an unlimited number of viewers. Each channel has one or more administrators who broadcasts public messages to the channel. Once someone joins a channel they have access to the entire message history. Iranian immigration-related channels include visa types and green card applications, immigration institutions, study abroad, lottery winners, exchanging money,

information about cities and states in the USA, Iranians in the US, as well as information specifically related to the exams like GRE. The number of members in these groups ranges from 500 to 45,000 . Messages in the channel typically include news-worthy updates and answers to frequently asked questions.

\section{Supergroups}

Telegram users use groups and 'supergroups' to connect with each other, have conversations about immigration topics, and ask questions. Supergroups, which can handle up to 100,000 members are used by Iranian immigrants to discuss topics of broad interest.

Controlling the shared information in the supergroups is challenging. Only admins can delete others' messages, and with some immigration supergroups reaching 45,000 members, controlling the information being shared in the supergroups requires constant effort and social policing. Supergroups allow for more flexibility than channels. Another feature of supergroups is that users can easily find and send direct messages to any other group member. Considering that members live in different countries, people may send messages at inconvenient times. As a drastic solution, some groups we observed used bots to temporarily deactivate themselves (Textbox 1). Direct message spam can also cause problems. Managing spam and fraudulent direct messages were frequent concerns in the supergroups we observed. Finally, for a group engaged in politically sensitive activities such as immigration, discoverability has potentially grave consequences.

\section{Links}

Direct links are the primary group discovery mechanism in Telegram. Some groups and channels are searchable if users know the exact term, but not all groups are easily found this way. In these instances, users must locate an out-of-band link to the channel or group, which has been posted online, or they must be given the link from friends or families. While this helps to keep the channels and groups private, it also makes it challenging for users to find groups of interest. This process is complicated by the social rules surrounding linking. Many groups and supergroups we observed explicitly prohibited link sharing (Textbox 2 ). If someone ignores these rules and shares the link to another group without prior permission, the admin can restrict or block the person immediately (Textbox 3 ).

On the surface, such practices may seem counterproductive, since links are the only way to find groups in the first place. In our observations, we encountered two main reasons. First, immigration supergroups are competitive in terms of the number of the members and their content. Even in cases when sharing links is allowed, members can request for links to other groups, but they need to ask for the links to be sent as a message in their private chat and not in the group (Textbox 4). The advantage to this is that users can talk to the person they are sharing the link with and ask for their purpose of joining the group. This increases privacy when they want to authorize someone to join. But this can make finding groups very 


After blocking the violator,
the admin posts a
screenshot of the
misconduct and explain
the reason of being
restricted in the caption:
"sharing unrelated content
with groups and without
coordination is prohibited.
User has become restricted
for 2 weeks."
A link to the group named
rules of the immigration
groups is added to this
message so that people
can join and learn more
about the details.

Textbox 3: This is an example of applying the rules on someone who shared a link to a research survey without prior notice to the admins in a group related to study in the US.

\section{A rule in a supergroup:}

"You want a specific group link. Write: Please send the link to the group $X$ in PV."

Textbox 4: An instance of the rules around link sharing in group related to study in the US. difficult since links and invitations are the only ways of finding and joining groups.

\section{Private channels and groups}

Telegram does provide a privacy-focused option for conversations: private channels and groups. Most of the time, private groups have fewer members.

Examples of these groups might be for people in a particular city or state in the USA to plan short trips to another city, to connect with other Iranians living in a special city, to buy traditional food. In future work, we plan to study private channels and groups more closely.

\section{Future work}

This work is part of an ongoing research study examining Iranian immigrants' online practices. In our future work, we will continue to examine the role of Telegram throughout the immigration stages, study users' appropriation of Telegram's features to meet their needs, and provide implications for the design of social support technologies for immigration.

\section{Conclusion}

Telegram is a messaging application and its platform is not well adapted for managing immigration practices as other tools like forums or task management applications. Through our observations on 30 immigration-related groups and channels on telegram, we examined how Iranians are using different features of Telegram to adapt it for their immigration management and the workarounds they have developed to overcome the limitations of the technology. We categorized and provided instances of such activities in this paper.

\section{References}

1. Caidi, N., Allard, D., \& Quirke, L. (2010).

Information practices of immigrants. Annual Review of Information Science and Technology, 44(1), 491-531.

https://doi.org/10.1002/aris.2010.1440440118

2. Dargahi Nobari, A., Reshadatmand, N., \& Neshati, M. (2017). Analysis of Telegram, An Instant Messaging Service. In Proceedings of the 2017 ACM on Conference on Information and Knowledge Management (pp. 2035-2038). New York, NY, USA: ACM. https://doi.org/10.1145/3132847.3133132

3. What Is Telegram, and Why Are Iran and Russia Trying to Ban It? - The New York Times. (n.d.), from

https://www.nytimes.com/2018/05/02/world/europ e/telegram-iran-russia.html

4. Using Social Network of TELEGRAM for Education on Continued Breastfeeding and Complementary Feeding of Children among Mothers: a Successful Experience from Iran. (n.d.). from http://ijp.mums.ac.ir/m/article_8570.html

5. Xodabande, I. (2017). The effectiveness of social media network telegram in teaching English language pronunciation to Iranian EFL learners. Cogent Education, 4(1), 1347081. https://doi.org/10.1080/2331186X.2017.1347081

6. The information needs and information seeking behaviour of immigrant southern Sudanese youth in the city of London, Ontario: an exploratory study | Library Review | Vol 55, No 4. (n.d.). https://www.emeraldinsight.com/doi/abs/10.1108/ 00242530610660807 